\title{
RELAÇÕES ÁGUA-SOLO-PLANTA-ATMOSFERA
}

\author{
DESEMPENHO DE UM SISTEMA DE IRRIGAÇÃO POR \\ MICROASPERSÃO, INSTALADO A NÍVEL DE CAMPO ${ }^{1}$
}

\author{
Antônio de Almeida Barreto Filho², José Dantas Neto ${ }^{3}$, Jose Arimatéia de Matos $^{4}$ \& \\ Everaldo Mariano Gomes ${ }^{5}$
}

\begin{abstract}
RESUMO
Este trabalho foi desenvolvido na Escola Agrotécnica Federal de Sousa, PB, com a finalidade de se avaliar o desempenho de um sistema de irrigação localizada tipo microaspersão. Avaliaram-se, em três subunidades deste projeto (A, B e C) a variação de pressão e de vazão, os coeficientes de uniformidade e a eficiência de aplicação. Foram observadas elevadas variações de vazão e de pressão em duas subunidades avaliadas. A uniformidade de irrigação apresentou resultados elevados, sendo classificado como excelente; por outro lado a eficiência de aplicação de água nas subunidades $\mathrm{A} \mathrm{e} \mathrm{C}$ foi aceitável, enquanto na subunidade B foi menor que o mínimo recomendável, que seria de $80,0 \%$, para microaspersão, fato que pode ser atribuído as elevadas variações absolutas de pressão e de vazão observadas em todas as laterais desta subunidade.
\end{abstract}

Palavras-chave: pressão, vazão, uniformidade, eficiência

FIELD PERFORMANCE OF A MICROSPRINKLER SYSTEM

\begin{abstract}
This work was conducted in the 'Escola Agrotécnica Federal de Sousa, PB', with the purpose of evaluating the performance of the microsprinkler system of localized irrigation. Three sub units of this project, (A, B and C), were evaluated for the variation of pressure and flow, coefficients of uniformity and the application efficiency. High flow and pressure variations were observed in two sub units appraised. The irrigation uniformity was high, being classified as excellent. The efficiency of application of water in the sub units $\mathrm{A}$ and $\mathrm{C}$ was acceptable, while in the area B it was less than the minimum recommendable of $80.0 \%$ for microsprinkler, a fact that may be attributed to the high absolute variations of pressure and of flow observed in all the lateral subunits.
\end{abstract}

Key words: pressure, flow, uniformity, efficiency

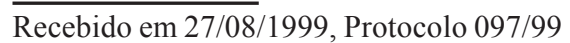

${ }^{1}$ Parte da Dissertação de Mestrado apresentada pelo primeiro autor à UFPB

${ }^{2}$ Eng. Agrícola, Mestre em Engenharia Agrícola - Irrigação e Drenagem, DEAg/CCT/UFPB, Av. Aprígio Veloso 882, Bodocongó, CEP 58109 - 970, Campina Grande, PB. Fone: (0xx83) 3333121

${ }^{3}$ Prof. Adjunto, Doutor, DEAg-CCT-UFPB. Fone: (0xx83) 310 1318, Fax: (0xx83) 310 1185. E-mail: zedantas@deag.ufpb.br

${ }^{4}$ Eng. Agrícola, Doutor em Irrigação e Drenagem, DEAg/CCT/UFPB. Fone: (0xx83) 310 1318, Fax: (0xx83) 3101185. E-mail:jamatos@deag.ufpb.br

${ }^{5}$ Eng. Agrônomo, Mestre em Engenharia Agrícola, Prof. da Escola Agrotécnica Federal de Sousa, Rua Tancredo Neves, s/n, Jardim Sorrilândia, CP 49, CEP 58805 - 970, Sousa, PB. Fone: (0xx83) 522 2727, Fax: (0xx83) 5222728 


\section{INTRODUÇÃO}

A utilização de sistemas de irrigação mais eficientes é uma busca constante na agricultura irrigada, pois existe tendência de aumento no custo da energia e de redução da disponibilidade hídrica dos mananciais. Dentre os sistemas pressurizados, a irrigação localizada é a que propicia a maior eficiência de irrigação, uma vez que as perdas na aplicação de água são relativamente pequenas, considerando-se que, quando bem projetada e manejada, a área máxima molhada não deve ser superior a $55 \%$ da área sombreada pela planta, com área mínima molhada de $20 \%$ nas regiões úmidas e de $30 \%$ nas regiões de clima semi-árido (Azevedo, 1986).

A irrigação por microaspersão e por gotejamento constitui os principais sistemas de irrigação localizada. O sistema microaspersão é caracterizado por apresentar pressão operacional menor que $207 \mathrm{kPa}$, vazão de $20 \mathrm{a} 100 \mathrm{~L} \mathrm{~h}^{-1}$ e diâmetro de alcance dos emissores variando de 1,5 a $10 \mathrm{~m}$ (Boman 1989).

Keller \& Bliesner (1990) comentam que é recomendável, após a instalação de um sistema de irrigação, proceder-se a testes de campo, com objetivo de se verificar a adequação da irrigação recomendando, quando necessário, ajustes na operação e, principalmente, no manejo. Esses procedimentos visam maximizar a eficiência do sistema.

Para Bernardo (1995) a eficiência de aplicação é a estimativa da percentagem do total de água aplicada na irrigação considerada útil às plantas. Em geral, a baixa eficiência nos projetos de irrigação está relacionada à desuniformidade de distribuição d'água. Segundo Frizzone (1992) a eficiência de aplicação incorpora a eficiência de distribuição e a eficiência em potencial de aplicação, dando idéia das perdas de água por percolação e por evaporação. Dentre os sistemas de irrigação, a localizada apresenta maiores valores de efíciência de aplicação, da ordem de 80 a $90 \%$, bem superiores às faixas de 60 a $80 \%$, e de 50 a $70 \%$, dos sistemas por aspersão e superfície, respectivamente (Keller \& Bliesner 1990).

A uniformidade da irrigação tem efeito no rendimento das culturas e é considerada um dos fatores mais importantes no dimensionamento e na operação de sistemas de irrigação. $\mathrm{O}$ conceito de uniformidade de distribuição foi introduzido por Christiansen em 1942, referindo-se à variabilidade da lâmina de água aplicada ao longo da extensão da superfície do terreno irrigado. A uniformidade de distribuição de água apresenta, em qualquer sistema de irrigação, importantes conseqüências na economia do projeto. Reduzidos valores de uniformidade determinam, em geral, maior consumo de água e energia, maior perda de nutrientes e, ao mesmo tempo, podem proporcionar plantas com déficits hídricos, em significativa proporção da área irrigada (Scaloppi \& Dias, 1996).

Segundo Boman (1989) a uniformidade é um indicador da igualdade (ou desigualdade) das taxas de aplicação dentro do diâmetro padrão de um emissor. Uniformidade alta é importante para irrigação em terras arenosas, onde a redistribuição lateral da água é limitada. Excessos de aplicação de água nesses solos resultam freqüentemente em lâmina percolada e lixiviação de nutrientes para fora da zona radicular. A uniformidade elevada pode ser especialmente desejável para fertirrigação ou quimirrigação, visto que as aplicações de nutrientes ou compostos químicos não serão mais uniformes que a distribuição de água.
A baixa uniformidade de distribuição de água pode ser atribuída a muitas causas. Para efeito de avaliação de sistemas já instalados, Bralts \& Kesner (1983) agrupam as causas em duas classes: a) em causas hidráulicas - todas aquelas que afetam a pressão de operação dos emissores, podendo ser oriundas de um projeto hidraulicamente mal concebido, da falta de reguladores de pressão ou desajuste desses reguladores, elevada perda de carga, elevado desnível geométrico etc.; b) em baixa uniformidade dos emissores - decorrente do alto coeficiente de variação de fabricação e/ou da obstrução dos emissores.

Conforme Abreu et al. (1987) a desuniformidade de aplicação nos sistemas localizados resulta dos seguintes fatores: das diferenças de pressão que se produzem na rede, devido às perdas de carga e à irregularidade da topografia do terreno; da insatisfatória uniformidade de fabricação dos emissores, em razão do inadequado controle de qualidade; do número de emissores por planta; da variação das características hidráulicas do emissor, ao longo do tempo, devido a possíveis obstruções e/ou envelhecimento; da temperatura; do efeito do vento, quando se usa microaspersão e da variação de fabricação dos reguladores de pressão, quando existirem.

Matos et al. (1996) dizem que vários são os fatores que influenciam na uniformidade de distribuição de água do microaspersor, podendo-se citar: posição do suporte, ângulo de irrigação, tronco e folhagem da árvore, elemento distribuidor, pressão de trabalho, reguladores e amortecedores e vazão nominal.

O objetivo deste trabalho foi avaliar o desempenho de um sistema de irrigação por microaspersão em nível de campo, numa área da Escola Agrotécnica Federal de Sousa, localizada dentro do Perímetro Irrigado de São Gonçalo, PB. Especificamente, determinou-se: variação de pressão $(\mathrm{P})$ e de vazão $(\mathrm{Q})$ nas tubulações laterais e sub-unidades de irrigação, além de coeficientes de uniformidade e eficiência de aplicação.

\section{MATERIAL E MÉTODOS}

Este trabalho foi realizado no Perímetro Irrigado de São Gonçalo, no município de Sousa, PB, cujas coordenadas geográficas são $6^{\circ} 45^{\prime} \mathrm{S}$ de latitude, $38^{\circ} 13^{\prime} \mathrm{W}$ de longitude e altitude de $233 \mathrm{~m}$. Segundo a classificação de Koppen, o clima é do tipo BSh, isto é, semi-árido quente. A temperatura média anual é de $27,8^{\circ} \mathrm{C}$, com precipitação média anual de $894 \mathrm{~mm}$, concentrada nos meses de janeiro a maio. A umidade relativa média do ar é de $58 \%$ e a velocidade média do vento é de $2,5 \mathrm{~m} \mathrm{~s}^{-1}$.

Numa área pertencente à Escola Agrotécnica Federal de Sousa (EAFS) localizada no perímetro, foram avaliadas três subunidades irrigadas por microaspersão, em uma área de aproximadamente 1,2 ha. As culturas implantadas nessas subunidades eram: subunidade "A", cultura de uva; "B", cultura de manga e "C", cultura de graviola, com os seguintes espaçamentos: $2,5 \times 4,0 \mathrm{~m}, 8,0 \times 8,0 \mathrm{~m}$ e $6,0 \times 5,0 \mathrm{~m}$, respectivamente.

As linhas de emissores de polietileno tinham $60 \mathrm{~m}$ de comprimento e diâmetro de $16 \mathrm{~mm}$ e as linhas secundária e principal de PVC, diâmetro de $50 \mathrm{~mm}$. O sistema era acionado por dois conjuntos motobombas de 2,0 CV cada um. 
As vazões foram medidas, no início, a um terço, a dois terços e no final de cada linha lateral e em quatro posições localizadas no início, a um terço, a dois terços e no final de cada linha de derivação nas subunidades escolhidas. Para determinação da vazão de cada microaspersor foi colocada, sobre esses, uma cuba capaz de interceptar o jato d'água que era coletado através de um pluviômetro e, em seguida, colocado em uma proveta graduada de $1.000 \mathrm{~mL}$. O tempo de duração para cada coleta foi de $1 \mathrm{~min}$, tempo este suficiente para se coletar volume superior a $200 \mathrm{~mL}$, valor mínimo recomendado pela ABNT (1987). Os volumes coletados foram convertidos em vazões $\left(\mathrm{L} \mathrm{h}^{-1}\right)$ cujo resultado é a média de três repetições.

Procedeu-se também às medidas de pressão, realizadas através de um manômetro de mercúrio, com escala de $0,66 \mathrm{kPa}$ (inferior aos $2 \mathrm{kPa}$ exigidos pela $\mathrm{ABNT}$, 1987) conectado ao tubo de conexão ou espaguete, distante $30 \mathrm{~cm}$ do emissor.

Foram determinadas as variações médias de pressão e vazão ao longo das linhas laterais, nas secundárias e nas subunidades, utilizando-se as seguintes equações:

$$
\begin{aligned}
& \Delta \mathrm{Q}=\frac{\text { Qmáx }- \text { Qmín }}{\text { Qmáx }} * 100 \\
& \Delta \mathrm{P}=\frac{\text { Pmáx }- \text { Pmín }}{\text { Pmáx }} * 100
\end{aligned}
$$

em que :

$$
\begin{array}{ll}
\Delta \mathrm{P} & \text { - variação de pressão, } \% \\
\text { Pmáx } & \text { - valor máximo de pressão, } \mathrm{kPa} \\
\text { Pmín } & \text { - valor mínimo de pressão, } \mathrm{kPa} \\
\Delta \mathrm{Q} & \text { - variação de vazão na lateral, } \% \\
\text { Qmáx } & \text { - valor máximo de vazão, } \mathrm{L} \mathrm{h}^{-1} \\
\text { Qmín } & \text { - valor mínimo de vazão, } \mathrm{L} \mathrm{h}^{-1} .
\end{array}
$$

Para se relacionar a variação de vazão com a variação de pressão aplicada, utilizou-se a equação proposta por $\mathrm{Wu} \&$ Yue (1993):

$$
\Delta \mathrm{Q}=1-(1-\Delta \mathrm{P})^{\mathrm{X} *} 100
$$

em que:

$\Delta \mathrm{Q}$ - variação de vazão na subunidade

$\Delta \mathrm{P}$ - variação de pressão

$\mathrm{X}$ - expoente de descarga do emissor.

A partir dos resultados de vazões encontrados em cada subunidade, determinou-se a uniformidade de irrigação, segundo as metodologias:

a) Metodologia de Merrian \& Keller (1978)

$$
\mathrm{CU}=100\left(\frac{\mathrm{q}_{25 \%}}{\mathrm{q}_{\text {med }}}\right)
$$

em que:

CU - coeficiente de uniformidade de irrigação da subunidade avaliada em campo, $\%$

$\mathrm{q}_{25 \%}$ - média de $25 \%$ do total de microaspersores com as menores vazões $\left(\mathrm{L} \mathrm{h}^{-1}\right)$ $\mathrm{q}_{\text {med }}$ - média das vazões coletadas de todos os microaspersores na subunidade $\left(\mathrm{L} \mathrm{h}^{-1}\right)$.

Com os dados de pressão obtidos nas subunidades, determinou-se o coeficiente de uniformidade de pressão:

$$
\mathrm{CUp}=100 *\left(\frac{\mathrm{P}_{25 \%}}{\mathrm{PM}}\right)^{\mathrm{X}}
$$

sendo:

CUp - coeficiente de uniformidade de pressão, \%

$\mathrm{PM}$ - pressão média da subunidade $(\mathrm{kPa})$

$\mathrm{P}_{25 \%}$ - pressão média de $25 \%$ das pressões mais baixas dentro da subunidade avaliada, $\mathrm{kPa}$

x - expoente de descarga do emissor.

A partir dos dados de pressão mínima, obtida na unidade operacional de irrigação que continha a subunidade, calculou-se a média e, em seguida, determinou-se o fator de correção das vazões, mediante a seguinte equação:

$$
\mathrm{FCV}=\left(\frac{\mathrm{P}_{25 \%}}{\mathrm{PM}_{\text {mín }}}\right)^{\mathrm{X}}
$$

em que:

FCV - fator de correção das vazões

$\mathrm{PM}_{\min }$ - média de todas as pressões mínimas das subunidades da unidade operacional de irrigação, $\mathrm{kPa}$

$\mathrm{P}_{25 \%}$ - pressão média de $25 \%$ das pressões mais baixas dentro das mínimas da subunidade avaliada, $\mathrm{kPa}$

$\mathrm{x} \quad$ - expoente de descarga do emissor.

Com os dados de vazão e suas respectivas pressões dos emissores ensaiados, determinou-se o valor do expoente de descarga através da seguinte expressão:

$$
\mathrm{x}=\frac{\log \left(\overline{\mathrm{q}}_{1} / \overline{\mathrm{q}}_{2}\right)}{\log \left(\mathrm{h}_{1} / \mathrm{h}_{2}\right)}
$$

em que:

$\overline{\mathrm{q}}_{1} \quad$ - a média de quatro emissores obtidos com a pressão $h_{1}$

$\overline{\mathrm{q}}_{2} \quad$ - a média de quatro emissores obtidos com a pressão $h_{2}$.

Nas subunidades estudadas " $A$ ", "B" e "C" encontraram-se, para $\mathrm{x}$, respectivamente, os valores 0,$32 ; 0,59$ e 0,43 .

A uniformidade de irrigação do sistema (CUS) é dada pela expressão:

$$
\mathrm{CUS}=\mathrm{CU} * \mathrm{FCV}
$$

A eficiência de aplicação (EA) sob irrigação completa foi estimada por Merrian \& Keller (1978):

$$
\mathrm{EA}=0,9 * \mathrm{CU}
$$


b) Metodologia da ASAE (1996)

A avaliação da uniformidade de irrigação, segundo a ASAE (1996) é baseada no método de estimação de uniformidade em campo, proposto por Bralts \& Kesner (1983) fundamentado no coeficiente de uniformidade estatístico. Selecionou-se uma subunidade de irrigação que estava funcionando em concordância com as condições normais de trabalho, avaliando-se a uniformidade de aplicação de água nas subunidades, a partir da equação:

$$
\mathrm{CUE}=(1-\mathrm{CVT}) * 100
$$

em que:

CUE - coeficiente de uniformidade estatístico de irrigação

CVT - coeficiente de variação total de vazão na subunidade.

Para a determinação do CVT na sub unidade, usou-se a seguinte equação:

$$
\mathrm{CVT}=\frac{\mathrm{S}_{\mathrm{q}}}{\mathrm{q}_{\mathrm{med}}}
$$

sendo:

$\mathrm{S}_{\mathrm{q}} \quad$ - desvio-padrão da vazão dos emissores $\left(\mathrm{L} \mathrm{h}^{-1}\right)$

qmed - vazão média dos emissores na subunidade $\left(\mathrm{L} \mathrm{h}^{-1}\right)$.

Com as pressões medidas na subunidade, obteve-se um coeficiente de variação da vazão por causas hidráulicas $(\mathrm{CVH})$ pela expressão:

$$
\mathrm{CVH}=\frac{\mathrm{S}_{\mathrm{h}}}{\mathrm{h}_{\mathrm{med}}}
$$

em que:

$\mathrm{S}_{\mathrm{h}}$ - desvio padrão das pressões medidas $(\mathrm{kPa})$

$\mathrm{h}_{\text {med }}$ - pressão média dos emissores na subunidade $(\mathrm{kPa})$.

O coeficiente de variação estatístico da vazão (CVE) foi determinado mediante:

$$
\mathrm{CVE}=\sqrt{\mathrm{CVT}^{2}-\mathrm{x}^{2} * \mathrm{CVH}^{2}}
$$

Para verificação do grau de aceitabilidade usou-se o procedimento de comparação do CU e do CUE, estabelecido pela ASAE (1996) segundo a Tabela 1.

Tabela 1. Comparação de uniformidades, $\%$

\begin{tabular}{lcc}
\hline Grau de Aceitabilidade & CUE & CU \\
\hline Excelente & $100-95$ & $100-94$ \\
Bom & $90-85$ & $87-81$ \\
Normal & $80-75$ & $75-68$ \\
Ruim & $70-65$ & $62-56$ \\
Inaceitável & $<60$ & $<50$ \\
\hline Fonte: ASAE EP458(1996) & &
\end{tabular}

\section{RESULTADOS E DISCUSSÃO}

Os resultados obtidos de pressões e vazões médias nas subunidades avaliadas encontram-se na Tabela 2. Observaram-se baixas pressões em todas as subunidades, principalmente nas "A" e "C", com valores abaixo da pressão de serviço recomendada pelos fabricantes para o sistema de microaspersão, que normalmente variam de 150 ou $200 \mathrm{kPa}$.

Tabela 2. Comportamento da pressão média (P) e da vazão (Q) ao longo das laterais, linhas de derivação e ao longo das subunidades irrigadas por microaspersão

\begin{tabular}{lrrr}
\hline & A & \multicolumn{1}{c}{$\mathrm{B}$} & \multicolumn{1}{c}{$\mathrm{C}$} \\
\hline Pressão $(\mathrm{kPa})$ & 76,90 & 125,00 & 66,10 \\
Vazão $\left(\mathrm{L} \mathrm{h}^{-1}\right)$ & 20,40 & 23,90 & 16,50 \\
$\Delta \mathrm{P}$ na linha lateral $(\%)$ & 25,00 & 20,00 & 3,50 \\
$\Delta \mathrm{Q}$ na linha lateral $(\%)$ & 10,00 & 19,00 & 5,70 \\
$\Delta \mathrm{P}$ na linha de derivação $(\%)$ & 27,00 & 20,00 & 14,00 \\
$\Delta \mathrm{Q}$ na linha de derivação $(\%)$ & 18,50 & 10,50 & 13,60 \\
$\Delta \mathrm{P}$ na subunidade $(\%)$ & 45,50 & 25,00 & 14,00 \\
$\Delta \mathrm{Q}$ na subunidade $(\%)$ & 20,70 & 2,60 & 11,40 \\
\hline
\end{tabular}

Os baixos valores de pressão devem-se à potência fornecida pelo conjunto motobomba. No projeto original constava da instalação de um conjunto de $6 \mathrm{CV}$ e, no momento, se encontra disponível apenas $4 \mathrm{CV}$; há de se considerar, também, a impureza da água, que proporcionou variação de pressão no cabeçal de controle, de até $45,5 \%$.

O microaspersor MSIII-Grafite, avaliado na subunidade "A", é recomendado pelo fabricante para trabalhar com pressão nominal de $150 \mathrm{kPa}$, proporcionando uma vazão de $27 \mathrm{~L} \mathrm{~h}^{-1}$; o valor médio da vazão encontrada foi, portanto, $32 \%$ menor que a recomendada pelo fabricante.

Encontra-se, ainda na Tabela 2, a dispersão absoluta da pressão e da vazão determinada através da variação de pressão $(\Delta \mathrm{P})$ e de vazão $(\Delta \mathrm{Q})$ ao longo das linhas laterais, das linhas de derivação e ao longo das subunidades irrigadas. Observou-se valores elevados de variação de pressão e de vazão ao longo das linhas laterais principalmente nas linhas utilizadas nas subunidades "A" e "B". Keller \& Karmeli (1974) recomendam que a variação de pressão ao longo da linha lateral deve ser de no máximo $11 \%$, conforme a Tabela 1 , sendo que apenas as linhas da subunidade "C" atenderam a essas recomendações. Esses valores são atribuídos à pressão de serviço dos emissores, diferente da recomendada pelo fabricante, além de serem encontradas em algumas linhas hastes sem emissores. Considera-se, também, a variação de pressão no cabeçal de controle, cujos valores chegaram a 45,5\%, devido a entupimentos nos filtros durante a realização dos testes.

Valores elevados também foram encontrados por Camp et al. (1997) trabalhando em sistemas de irrigação por gotejamento, com tubos novos e usados. Os autores em laboratório encontraram variação de vazão em tubos novos de 5,2\%; em tubos usados em campo, a variação de vazão atingiu $29,5 \%$. As linhas de gotejadores, quando enterradas, proporcionaram variação de vazão de até $92,6 \%$, devido a problemas de obstrução dos emissores.

Nas linhas de derivação nas subunidades avaliadas, a variação de pressão foi de até $27 \%$, quando a variação máxima 
recomendada é $9 \%$; já dentro de cada subunidade, a variação de pressão foi de até $45,5 \%$. Keller \& Karmeli (1974) recomendam uma variação de pressão de até $20 \%$ na subunidade; na Tabela 2 observa-se que apenas a subunidade " $\mathrm{C}$ " atende a essa exigência.

Considerando-se que o valor do expoente de descarga $\mathrm{x}$ nas subunidades estudadas "A", "B" e "C" foi de $0,32,0,59$ e 0,43 , respectivamente, a variação de vazão dada pela Eq. 3 deveria ser: 17,6; 15,6 e 6,3\%; entretanto, conforme se observa na Tabela 2 os valores encontrados sempre foram maiores. Keller \& Karmeli (1974) recomendam uma variação de vazão dos emissores extremos nessas subunidades de no máximo $10 \%$.

Não se observou, nas avaliações, escoamento superficial próximo aos emissores, mas as vazões coletadas junto às hastes dos microaspersores para todas as subunidades não ultrapassaram $0,1 \%$ das vazões coletadas nesses emissores. Este valor foi bem inferior ao valor máximo de $9 \%$, encontrado por Alves \& Lima (1994). Dantas Neto et al. (1997) observaram escoamento junto à haste dos microaspersores NAAN 7110, em condições de campo, de 0,31, 0,30, 0,28 e 0,24 $\left(\mathrm{L} \mathrm{h}^{-1}\right)$, o que corresponde a $0,80,7,0,7$ e $0,6 \%$ do volume aplicado, respectivamente. Rocha (1996) trabalhando com o microaspersor australiano ANTELCO, modelo RRN30, observou, durante os testes, que escorreu, junto à haste, $0,60 \mathrm{a}$ $0,74 \%$ do volume de água aplicado pelo microaspersor.

Nas subunidades estudadas os valores de CVH foram considerados altos, variando de 0,11 a 0,17 , enquanto o CVT varia de 0,08 a 0,11 , classificado como bom pela ABNT (1987). De acordo com Bralts \& Kesner (1983), numa linha lateral o coeficiente de variação de vazão dos emissores, resultantes apenas dos efeitos hidráulicos é, em geral, da ordem de 0,037 a 0,078 . Esses valores decorrem de variações de vazão de $10 \%$ a $20 \%$, normalmente utilizados em projetos de irrigação. Zanini et al. (1998) avaliando um sistema de irrigação por microaspersão em cultura de bananeira, encontraram coeficiente de variação de vazão total de 0,85 a 0,11 .

Conforme a Tabela 3, o coeficiente de variação estatístico do emissor (CVE) que indica a variação da vazão devido a causas diferentes da hidráulica (principalmente variabilidade de fabricação, obstruções do emissor, tempo de uso e sustentabilidade de variação da vazão com o tempo) variou de 0,02 a 0,06 ; esses valores são considerados baixos, caracterizando bom coeficiente de variação de fabricação dos emissores analisados, e, com o pouco tempo de uso não ocorreu entupimento. Segundo normas da ASAE (1996) quando ocorre obstrução dos emissores o CVE apresenta-se excessivamente alto, com valores acima de 0,20 ; portanto, nas subunidades estudadas as variações de vazão são devidas principalmente às variações de pressão.

Tabela 3. Resultado da uniformidade de irrigação e da eficiência de aplicação das subunidades do sistema

\begin{tabular}{lccc}
\hline \multicolumn{1}{c}{ Índices } & \multicolumn{3}{c}{ Subunidades } \\
\cline { 2 - 4 } Avaliados & $\mathrm{A}$ & $\mathrm{B}$ & $\mathrm{C}$ \\
\hline CVH & 0,17 & 0,19 & 0,11 \\
CVT & 0,09 & 0,11 & 0,08 \\
CVE & 0,02 & 0,05 & 0,06 \\
CU (\%) & 89,10 & 87,00 & 94,20 \\
CUP (\%) & 91,30 & 86,60 & 83,69 \\
CUE (\%) & $91,00 \pm 3,23$ & $89,00 \pm 4,01$ & $92,00 \pm 2,83$ \\
EA (\%) & 80,10 & 78,30 & 84,60 \\
\hline
\end{tabular}

Os valores dos coeficientes de uniformidade de irrigação (CU) e os de uniformidade estatística (CUE) das subunidades avaliadas em campo foram elevados, sendo o $\mathrm{CU}$ da subunidade "A" considerado excelente, segundo normas da ASAE (1996) enquanto os demais foram considerados de boa aceitabilidade; podendo-se inferir, então, que o projeto foi hidraulicamente bem dimensionado e que os emissores são de boa qualidade. A ABNT (1987) recomenda valores de uniformidade de emissão (CU) de 75 a $80 \%$ para microaspersão em topografia uniforme e gradiente de declive inferior a $\%$, enquanto Abreu et al. (1987) consideram, dentro dos limites aceitáveis de funcionamento, valores de CU acima de 80\%. Sousa et al. (1998) trabalhando com sistema de microaspersão instalado numa área cultivada com banana encontraram coeficiente de uniformidade médio de $82 \%$, enquanto Almeida (1997) encontrou coeficientes de uniformidade elevados, variando de 91,2 a $97 \%$.

Os valores do coeficiente de uniformidade de pressão (CUP) podem ser considerados elevados, já que variaram de $83,5 \%$ a 93,8\%; um limite de valor mínimo recomendado para este coeficiente é de $80 \%$.

$\mathrm{Na}$ Tabela 2, os valores de eficiência de aplicação (EA) para as subunidades estudadas, variaram de 78,3 a $84,6 \%$. Keller \& Bliesner (1990) recomendam valores em torno de $80 \%$. Considerando-se que mesmo num sistema de irrigação bem manejado podem ocorrer perdas por percolação profunda de até $10 \%$, os resultados encontrados são considerados satisfatórios.

Conforme metodologia de Merrian \& Keller (1978) o expoente médio de descarga do emissor (x) encontrado nas subunidades estudadas foi igual a 0,45 e o fator médio de correção de vazão (FCV) foi igual a 1,06. Como o CU médio é de $90,1 \%$ a uniformidade de irrigação do sistema instalado (CUS) é de $95,5 \%$.

\section{CONCLUSÕES}

1. A pressão de operação dos emissores não está de acordo com a recomendada pelo fabricante.

2. A variação absoluta de pressão e de vazão nos extremos das subunidades irrigadas avaliadas apresentou-se elevada.

3. Os coeficientes de uniformidade de irrigação das subunidades avaliadas foram elevados nas diferentes metodologias propostas, mostrando que o sistema foi hidraulicamente bem dimensionado e utilizou emissores de boa qualidade.

4. O valor do índice de eficiência de aplicação nas subunidades foi considerado bom, caracterizando uma boa qualidade da irrigação.

5. Recomenda-se a instalação de um conjunto moto-bomba mais potente ou, então, instalar-se um conjunto paralelo, de maneira que aumente pelo menos em $2 \mathrm{CV}$ a potência total.

\section{AGRADECIMENTOS}

Os autores agradecem à Escola Agrotécnica Federal de Sousa (EAFS) Sousa, PB, pelo apoio e participação na presente pesquisa. 


\section{REFERÊNCIAS BIBLIOGRÁFICAS}

ABNT- ASSOCIAÇÃO BRASILEIRA DE NORMAS TÉCNICAS. Sistema de irrigação localizada: Avaliação do desempenho. Rio de Janeiro, 11p, 1987. Projeto 12: 02-08-20.

ABREU, J.M.H.; LOPEZ, J.R.; REGALADO, A.P.; HERNANDEZ, J.F.G. El riego localizado. Madrid: Instituto Nacional de Investigaciones Agrárias, 1987,317p.

ALMEIDA, F.T. Avaliação dos sistemas de irrigação pressurizados e do manejo da água na cultura da banana no Projeto Gorutuba. Viçosa: UFV, 1997. 96p. Dissertação Mestrado

ALVES, D.R.D.; LIMA, L.A. Avaliação da uniformidade de distribuição de água de microaspersores e microdifusores. In: CONGRESSO BRASILEIRO DE ENGENHARIA AGRÍCOLA, 23, 1994, Campinas. Resumos... Campinas: Sociedade Brasileira de Engenharia Agrícola, 1994, n.94-3 - 316. p. 17.

ASAE - AMERICAN SOCIETY OF AGRICULTURAL ENGINEERS. Field Evaluation of Microirrigation Systems. St. Joseph, p.792-797. 1996.

AZEVEDO H.M. Irrigação localizada. Informe Agropecuário, Belo Horizonte, v.12, n.139, p.40-53, 1986.

BERNARDO, S. Manual de irrigação. $6^{\mathrm{a}}$ ed. Viçosa: UFV, 1995. $657 \mathrm{p}$.

BOMAN, B.J. Distribution patterns of microirrigation spinner and spray emitters. Applied Engineering in Agriculture, St. Joseph v.5, n.2, p.50-56. 1989.

BRALTS, V.F.; KESNER, C. Drip irrigation field uniformity estimation. Transactions of the ASAE, St. Joseph, v.24, n.5, p.1369-1374. 1983.

CAMP, C.R.; SADLER, E.J.; BUSSCHER, W.J. A comparision of uniformity measures for drip irrigation systems. Transactions of the ASAE, St. Joseph, v.40, n.4, p.1013-1020. 1997.

DANTAS NETO, J.; MEDEIROS, M.G.A.; AZEVEDO, C.A.V.; AZEVEDO, H.M. Performance hidráulica e perfil de distribuição de água do microaspersor NAAN 7110, sob diferentes condições de vento. Revista Brasileira de Engenharia Agrícola e Ambiental, Campina Grande, v.1, p.57-61. 1997.
FRIZZONE, J.A. Irrigação por aspersão: Uniformidade e eficiência. Piracicaba-ESALQ: Departamento de Engenharia Rural, 1992. 53p. Série Didática, 03

KELLER, J.; BLIESNER, R.D. Sprinkle and trickle irrigation. New York: van Nostrand Reinhold, 1990. 652p.

KELLER, J.; KARMELI, D. Trickle irrigation design parameters. Transactions of the ASAE, St. Joseph, v.17, n.4, p.678-684. 1974.

MATOS, J.A.; DANTAS NETO, J.; AZEVEDO, H.M.; AZEVEDO, C.A.V. Características hidráulicas do microaspersor Dan Sprinkler 2001. Irriga, Botucatu, v.1, n.3, p.30-44. 1996.

MERRIAN, J.L.; KELLER, J. Farm irrigation system evaluation: A guide for management. Logan: Agricultural and Irrigation Engineering Departament, Utah State University, 1978. 271p.

ROCHA, F.A. Características hidráulicas do microaspersor ANTELCO, modelo RRN30. In: CONGRESSO BRASILEIRO DE ENGENHARIA AGRÍCOLA, 26, 1996, Bauru. Resumos... Bauru: Sociedade Brasileira de Engenharia Agrícola, 1996. CDRom.

SCALOPPI, J.E.; DIAS, K.F.S. Relação entre a pressão de operação e a uniformidade de distribuição de água de aspersores rotativos por impacto. In: CONGRESSO BRASILEIRO DE ENGENHARIA AGRÍCOLA, 26, 1996, Bauru. Resumos... Bauru: Sociedade Brasileira de Engenharia Agrícola, 1996. CD Rom.

SOUSA, V.F.; FOLEGATTI, M.V.; ARAGÃO, E.C.; REBELO, A.L.F.; BASTOS, E.A.Uniformidade de emissão de água em um sistema de irrigação por microaspersão instalado numa área cultivada com banana. In: CONGRESSO BRASILEIRO DE ENGENHARIA AGRÍCOLA, 27, 1998, Poços de Caldas. Resumos... Poços de Caldas: Sociedade Brasileira de Engenharia Agrícola, 1998, v.1, p.211-213.

WU, I.P.; YUE, R. Drip lateral design using energy gradient line approach. Transactions of the ASAE, St. Joseph, v.36, n.2, p.389-394. 1993.

ZANINI, J. R.; PAVANI, L.C.; TAROZZO, M. Avaliação da vazão de um sistema de irrigação por microaspersão em cultura de bananeira. In: CONGRESSO BRASILEIRO DE ENGENHARIA AGRÍCOLA, 27, 1998, Poços de Caldas. Resumos... Poços de Caldas: Sociedade Brasileira de Engenharia Agrícola, 1998, v.2, p.290-292. 